\title{
Resonance Scan Simulation of Charmonium like states using PANDAROOT Framework
}

\section{Subodh S. Godre*i}

Department of Physics, Veer Narmad South Gujarat University, Surat - 395007, India

E-mail: ssgodre@yahoo.com

\section{Rajeshkumar Dudhat}

Department of Applied Physics, Sardar Vallabhbhai National Institute of Technology, Surat 395007, India

E-mail: raju_dudhateyahoo.co.in

\author{
Ajay Kumar Rai \\ Department of Applied Physics, Sardar Vallabhbhai National Institute of Technology, Surat - \\ 395007, India \\ E-mail: raiajayk@gmail.com
}

\section{on behalf of PANDA Collaboration}

Many Charmonium like exotic XYZ states have been observed in different experiments in recent years, some of these states are yet unexplained. Their correct theoretical interpretations require accurate determinations of their properties like mass and width. Future experimental facility PANDA (antiProton ANnihilation at DArmstad) @ FAIR involving a cooled antiproton beam will be well suited for better studies of these states. Width of these states can be well determined by energy resonance scan studies. Resonance scan simulation results for some states like $\mathrm{X}(3872), \mathrm{Y}(4140)$ and Y(4260) making use of the latest version of the PANDAROOT- MonteCarlo simulation framework for $\overline{\mathrm{P}} \mathrm{ANDA}$ experiment are presented.

XV International Conference on Hadron Spectroscopy-Hadron 2013

4-8 November 2013

Nara, Japan

\footnotetext{
${ }^{*}$ Speaker.

${ }^{\dagger}$ Parallel talk.
} 


\section{Introduction}

A number of charmonium like $\mathrm{X}, \mathrm{Y}, \mathrm{Z}$ states have been discovered in different experiments during past 10 years. Most of these can not fit into charmonium model and an interpretation of many states still remain unclear like X(3872), Y(4260), Y(4140) etc. These are associated with charmonium because they decay predominantly into charmonium states such as $J / \psi$, but their interpretation is far from obvious. For the proper understanding of XYZ states it would be necessary to study them with possible decay mode and get the solid knowledge of its properties like mass, width and decay fraction.

The PANDA experiment (antiProton ANnihilation at DArmstadt) is one of the key project under FAIR at GSI, Germany [1]. It will be using cooled and highly intense antiproton beam with momentum between 1.5 to $15 \mathrm{GeV}$, interacting with various internal targets. The PANDA detector is multi purpose detector, covering a $4 \pi$ solid angle. The goal of this experiment covers a wide range of high precision hadron spectroscopy, particular the search for exotic states in charmonium region. $\bar{p} p$ annihilations are particularly suitable for experimental studies of XYZ states, because resonances of all non-exotic quantum numbers can be formed directly whereas the formation of resonances with quantum numbers other than $J^{p c}=1^{--}$is suppressed in $e^{+} e^{-}$collisions.

We study resonance states by Monte Carlo (MC) simulation technique using the PANDAROOT framework - a software toolkit for PANDA experiment [2]. Physics events are generated using event generator EvtGen [3]. Generated particles are transported through the PANDA detector geometry using transport model Geant3/Geant4 [4], after that digitization, reconstruction, particle identification and analysis is carried out using ROOT [5]. The track finder and track fitter algorithms were used for pattern recognition to achieve a realistic signal reconstruction.

For study of resonance one can use the resonance scan technique and determine the resonance parameters with high precision. Using this technique we have carried out resonance scan simulation of X(3872), Y(4260) and Y(4140) states using PANDAROOT version 21620 with Geant3.

\section{Resonance Scan Technique}

In resonance scan technique, resonance parameters are measured by scanning the center of mass energy $\sqrt{s}$ across the resonance $\mathrm{R}$ by adjusting the beam momentum $p_{\text {beam }}$. The number of expected signal events $n\left(\sqrt{s_{0}}\right)$ for a beam energy $\sqrt{s_{0}}$ is calculated using formula:

$$
n\left(\sqrt{s_{0}}\right)=B R(R \rightarrow \text { final }) \cdot \mathscr{L} \cdot \alpha \cdot T \times \int \sigma_{B W}(\sqrt{s}) \cdot B\left(\sqrt{s}, \sqrt{s_{0}}\right) d \sqrt{s}
$$

where

$$
\begin{gathered}
\sigma_{B W}(\sqrt{s})=\frac{(2 J+1) \cdot 4 \pi \cdot \Gamma_{R}^{2}}{\sqrt{s^{2}-4 m_{p}^{2}}} \times \frac{B R(R \rightarrow p \bar{p}) \cdot B R(R \rightarrow \text { final })}{4\left(\sqrt{s^{2}-m_{R}}\right)^{2}+\Gamma_{R}^{2}} \\
B\left(\sqrt{s}, \sqrt{s_{0}}\right)=\frac{1}{\sqrt{2 \pi} \cdot \triangle \sqrt{s}} \cdot \exp \left(-\frac{\left(\sqrt{s}-\sqrt{s_{0}}\right)^{2}}{2(\triangle \sqrt{s})^{2}}\right)
\end{gathered}
$$

$\mathscr{L}$ - is integrated luminosity (0.864 $p b^{-1} /$ day - high resolution mode), $\varepsilon$ - efficiency (50\%), T beam time (1 day), $\sigma_{B W}$ - Breit-Wigner production cross section and $B\left(\sqrt{s}, \sqrt{s_{0}}\right)$ - distribution for nominal center of mass energy $\left(\Delta \sqrt{s} \simeq 33.568 \mathrm{KeV}\right.$ and $\left.\Delta p / p=2 \times 10^{-5}\right)$. Similarly background events were simulated separately from signal events, but analyzed with the same macros as the signal. The resonance scan energy range, number of energy scan points, energy interval between 


\begin{tabular}{|l|c|c|c|c|c|c|}
\hline Resonance State & Scan range $(\mathrm{GeV})$ & No of points & $\Delta E(\mathrm{MeV})$ & $\Gamma_{\text {in }}(\mathrm{MeV})$ & $\sigma_{\text {signal }}(\mathrm{nb})$ & $\sigma_{\text {bg }}(\mathrm{nb})$ \\
\hline \hline$X(3872) \rightarrow J / \psi \pi^{+} \pi^{-}$ & $3.87154-3.87249$ & 20 & 0.05 & 000.2 & 66.6 & 1.2 \\
\hline$Y(4260) \rightarrow J / \psi \pi^{+} \pi^{-}$ & $4.03400-4.48400$ & 20 & 22.0 & 108.0 & 01.9 & 5.5 \\
\hline$Y(4140) \rightarrow J / \psi \phi$ & $4.13000-4.17000$ & 40 & 01.0 & 011.7 & 19.0 & 2.1 \\
\hline $\mathrm{Y}(4140) \rightarrow J / \psi \omega$ & $4.09000-4.19000$ & 20 & 05.0 & 011.7 & 19.0 & 6.2 \\
\hline
\end{tabular}

Table 1: Parameters for resonance scan simulation.

these scan points $(\Delta E)$, the input width of the resonance $\left(\Gamma_{i n}\right)$, the production cross section $\left(\sigma_{\text {signal }}\right)$, and cross section for the background $\left(\sigma_{b g}\right)$ are given in the Table 1 for the resonance states studies in this work.

For each energy scan point, counts of reconstructed resonance candidates from signal and background events are added and the resulting counts are plotted against the respective nominal center of mass energy of the scan point. The excitation curve is a convolution of resonance cross section (Breit-Wigner) and the beam energy distribution (Gaussian). The mass and decay width of resonance are extracted by an analysis of the resulting curve. The event reconstruction and analysis methodology adopted in this work is similar to that given in detail in reference [6].

\section{3. $X(3872)$ state}

The X(3872) resonance was first observed by BELLE in study of the $\pi^{+} \pi^{-} J / \psi$-invariant mass distribution in exclusive $B^{+} \rightarrow K^{+} \pi^{+} \pi^{-} J / \psi$ decay channel [7]. It has been confirmed by CDF, D0, BABAR and LHCb experiments. The world average mass of this state is $M_{X(3872)}=3871.68$ $\pm 0.17 \mathrm{MeV}$, that is very close to the $m_{D^{0}}+m_{\bar{D}^{* 0}}$ threshold mass which predicts that the $\mathrm{X}(3872)$ might be a loosely bound $D^{0} \bar{D}^{* 0}$ molecular state; other possibilities are tetra-quark, a diquark antiquark bound state, hybrid charmonium or mixtures. Its quantum numbers is $J^{P C}=1^{++}$and the width $\Gamma_{X(3872)}$ is unknown, only an upper limit of $1.2 \mathrm{MeV}$ at $90 \%$ C.L. is published.

We have studied $\mathrm{X}(3872)$ state using resonance scan technique for signal channel: $\bar{p} p \rightarrow$ $X(3872) \rightarrow J / \psi \pi^{+} \pi^{-} \rightarrow e^{+} e^{-} \pi^{+} \pi^{-}$and for background channel: $\bar{p} p \rightarrow J / \psi \pi^{+} \pi^{-} \rightarrow e^{+} e^{-} \pi^{+} \pi^{-}$. In the analysis final stage particles $e^{ \pm}$and $\pi^{ \pm}$are identify from likelihood based selection algorithm. We have applied electron/pion discrimination to classify all $e^{ \pm}$and $\pi^{ \pm}$present in the events. All combinations of one electron and one positron candidate in the same event were used to calculate the two particle invariant mass. From $e^{ \pm}$pair we reconstruct $J / \psi$ mass and apply mass cut from $2.7 \mathrm{GeV}$ to $3.5 \mathrm{GeV}$ to remove the background. We have calculated missing mass for pion and if this value falls in $J / \psi$ mass region with mass range from $2.7 \mathrm{GeV}$ to $3.5 \mathrm{GeV}$ then it is selected as pion candidate. If the event contained one or more suitable $J / \psi$ candidates and at least one possible pion pair with a missing mass then this event is used to reconstruct $\mathrm{X}(3872)$ candidate. If this mass value falls in the interval from $3.6 \mathrm{GeV}$ to $4.0 \mathrm{GeV}$, the candidate counter for the scan point is raised by one. For cross section calculation (equation 2.2) the value of $B(X(3872) \rightarrow \bar{p} p)$ is taken from reference [8]. The background is presumed to be constant over the (narrow) width of the resonance. The excitation curve is shown in Figure 1(a) with the fitted value of the mass and width of the resonance for $\mathrm{X}(3872)$ state given in the inset. 

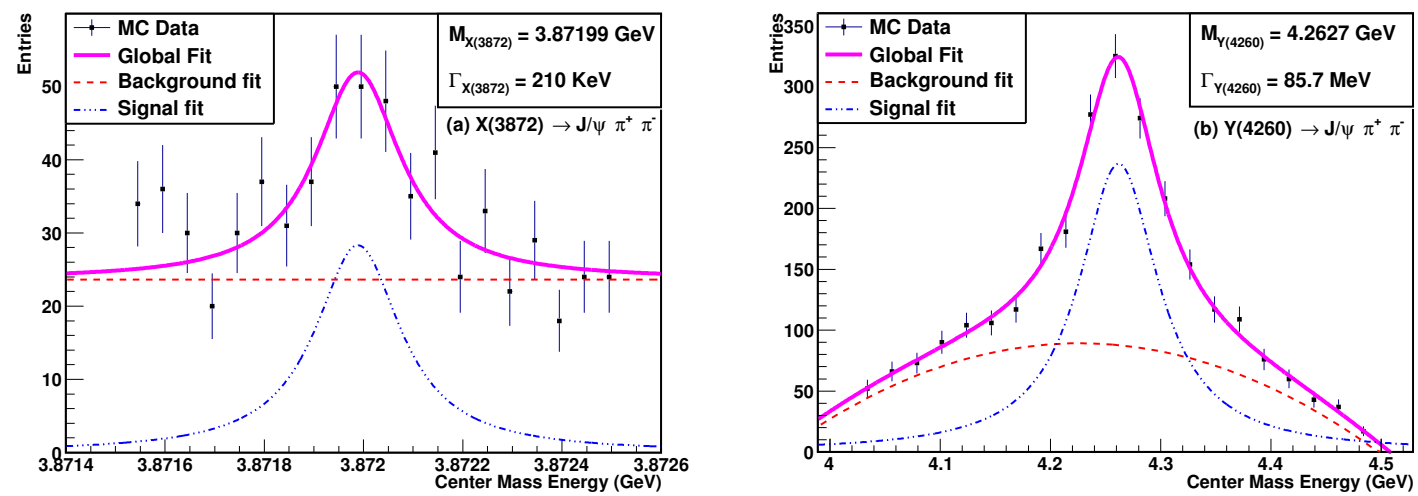

Figure 1: MC results using resonance scan technique: (a) $X(3872)$ state (b) $Y(4260)$ state.

\section{4. $Y(4260)$ state}

The exotic charmonium like state $\mathrm{Y}(4260)$ was first observed by the BABAR Collaboration in the initial state radiation process: $e^{+} e^{-} \rightarrow \gamma_{I S R} \pi^{+} \pi^{-} J / \psi$ [9]. It was confirmed by the CLEO and BELLE Collaboration. The PDG average mass of this state is $M_{Y(4260)}=4250 \pm 9 \mathrm{MeV}$, width $\Gamma_{Y(4260)}=108 \pm 12 \mathrm{MeV}$ and quantum numbers $J^{P C}=1^{--}$. Many suggestions have been made for its structure likes a tetra-quark state, a hadronic molecule, hybrid charmonium or charm baryonium.

We have studied Y(4260) resonance using signal channel: $\bar{p} p \rightarrow Y(4260) \rightarrow J / \psi \pi^{+} \pi^{-} \rightarrow$ $e^{+} e^{-} \pi^{+} \pi^{-}$and background channel: $\bar{p} p \rightarrow J / \psi \pi^{+} \pi^{-} \rightarrow e^{+} e^{-} \pi^{+} \pi^{-}$. For reconstruction of intermediate and final stage particles we use mass cuts similar to $X(3872)$ resonance case. If $Y(4260)$ candidate mass value falls in the interval from 3.8 to $4.6 \mathrm{GeV}$, the candidate counter for the scan point is raised by one. The background was assumed to be constant and the number of background events generated for all the energy scan points were the same. The excitation curve is shown in Figure 1(b) with the fitted value of the mass and width of the resonance for Y(4260) state given in the figure inset.

\section{5. $Y(4140)$ state}

The CDF Collaboration observed a narrow structure near threshold, termed as the $\mathrm{Y}(4140)$ meson, in the $J / \psi \phi$ mass spectrum in the exclusive $B^{+} \rightarrow J / \psi \phi K^{+}$decays with the mass $M_{Y(4140)}$ $=4143.0 \pm 2.9$ (stat) $\mathrm{MeV}$ and natural width $\Gamma_{Y(4140)}=11.7 \pm 8.3$ (stat) $\mathrm{MeV}$ [10]. Many possible alternative interpretations such as hadronic molecules, tetra quark states or even hybrid configurations have been proposed for structures of these states.

We have performed the simulation of the Y(4140) in two different possible decay modes:

1. Signal channel: $\bar{p} p \rightarrow Y(4140) \rightarrow J / \psi \phi \rightarrow e^{+} e^{-} K^{+} K^{-}$and background channel: $\bar{p} p \rightarrow$ $J / \psi \phi \rightarrow e^{+} e^{-} K^{+} K^{-}$.

2. Signal channel: $\bar{p} p \rightarrow Y(4140) \rightarrow J / \psi \omega \rightarrow e^{+} e^{-} \pi^{+} \pi^{-} \pi^{0}$ and background channel: $\bar{p} p \rightarrow$ $J / \psi \omega \rightarrow e^{+} e^{-} \pi^{+} \pi^{-} \pi^{0}\left(\pi^{0} \rightarrow \gamma \gamma\right)$.

In both cases analysis is carried out which is essentially similar to that in the case of X(3872) and $\mathrm{Y}(4260)$ case. First we reconstructed the final stage particles $e^{ \pm}, K^{ \pm}, \pi^{ \pm}$and $\gamma$; using these 

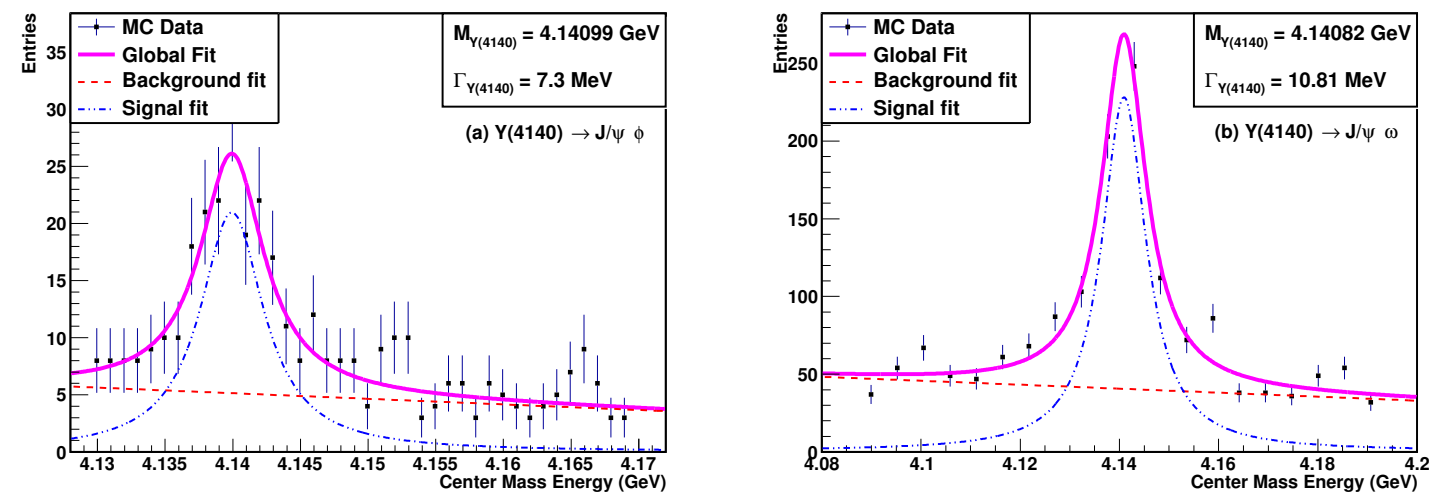

Figure 2: MC results using resonance scan technique (a) $Y(4140) \rightarrow J / \psi \phi$ (b) $Y(4140) \rightarrow J / \psi \omega$.

candidates one can reconstruct intermediate particles: $e^{+} e^{-} \rightarrow J / \psi, K^{+} K^{-} \rightarrow \phi, \gamma \gamma \rightarrow \pi^{0}$ and $\pi^{ \pm} \pi^{0} \rightarrow \omega$. Here we applied mass cut $(1.01,1.03) \mathrm{GeV}$ for $\phi$ and $(0.75,0.81) \mathrm{GeV}$ for $\omega$; these are used for resonance reconstruction: $J / \psi \phi \rightarrow Y(4140)$ and $J / \psi \omega \rightarrow Y(4140)$. If this mass value falls in the interval from $3.3 \mathrm{GeV}$ to $4.2 \mathrm{GeV}$, the candidate counter for the scan point is raised by one. The background was presumed to be constant over the width of the resonance. The excitation curve is shown in Figure 2(a) for $Y(4140) \rightarrow J / \psi \phi$ and (b) for $Y(4140) \rightarrow J / \psi \omega$ with the fitted value of the mass and width of the resonance for Y(4140) state given in the inset for the respective decay modes.

\section{Summary}

We have performed MC simulation for the X(3872), Y (4260) and Y(4140) resonance states using PANDAROOT framework and reconstructed masses and widths of these states using resonance scan technique. From this we can conclude that the PANDA experiment will be able to measure the width of $\mathrm{X}(3872)$ more precisely and it will be most suitable experimental set up for study of different properties of many unexplained resonance states.

\section{References}

[1] $\bar{P} A N D A$ Collabration, Physics Performance Report for P̄ANDA, (2009).

[2] Website: http://panda-wiki.gsi.de/cgi-bin/view/Computing/PandaRoot.

[3] D. J. Lange, Nucl. Instrum. Meth. A 462, (2001) 152 - 155.

[4] S. Agostinelli et al., Nucl. Instrum. Meth. A 506, (2003) 250.

[5] R. Brun and F. Rademakers, Phys. Res. A 389, (1997) 81-86.

[6] Martin J. Galuska et al., in proceedings of Bormio conference, PoS (Bormi 02012 ) 018.

[7] S.-K. Choi et al., (BELLE Collaboration), Phys. Rev. Lett. 91, (2003) 262001.

[8] R. Aaij et al., (LHCb Collaboration), Eur. Phy. J. C 73, (2013) 2462.

[9] B. Aubert et al.,(BABAR Collaboration), Phys. Rev. Lett. 95, (2005) 142001.

[10] T. Aaltonen et al., (CDF Collaboration), Phys. Rev. Lett. 102, (2009) 242002. 